\title{
ACTIVIDADES PARA EL APRENDIZAJE DEL INGLÉS COMO LENGUA EXTRANJERA EN LIBROS DE TEXTO DE PRIMARIA ${ }^{1}$
}

\author{
Joaquín Gris Roca \\ Universidad de Murcia \\ joaquingrisroca@gmail.com
}

\begin{abstract}
RESUMEN: Alcanzar la fluidez necesaria en las destrezas orales y escritas es una de las metas más importantes para los aprendices de Inglés como Lengua Extranjera (ILE). Las distintas actividades presentes en los libros de texto pueden tener diferentes potenciales de enseñanza explícita e implícita según el tipo de conocimiento que promuevan. Por tanto, el tipo de conocimiento que los aprendices logran va a depender, en gran medida, del énfasis que los profesores pongan en las distintas actividades. Dado que los aprendices jóvenes tienden a beneficiarse más de las actividades de carácter comunicativo, el presente estudio exploratorio tiene como objetivo examinar el potencial explícito e implícito de una muestra de 1.952 actividades extraídas de 10 libros de texto de primaria para la Enseñanza del ILE en España. Los resultados mostraron que la proporción de actividades dedicadas a la enseñanza de las formas es más elevada de lo esperable.
\end{abstract}

PALABRAS CLAVE: Escuela Primaria, Inglés como Lengua Extranjera, potencial de enseñanza explícita e implícita, corpus de actividades.

\section{LEARNING ACTIVITIES IN THE CONTEXT OF PRIMARY SCHOOL EFL TEXTBOOKS}

\begin{abstract}
Attaining the necessary fluency in the oral and written skills is one of the fundamental goals that EFL students need to pursue. Different activities in textbooks can have different explicit and implicit teaching loads depending on the type of knowledge promoted. Therefore, the kind of knowledge achieved by EFL learners might depend greatly on the typology of activities that teachers implement. Insofar as younger learners seem to benefit more from activities whose
\end{abstract}

1. Este trabajo contó con el apoyo del Ministerio de Economía, Industria y Competitividad (Referencia del proyecto de investigación FFI2013-44979-R). 
focus is communicative (hence, with a higher implicit teaching potential), the objective of the present exploratory study is to examine the explicit and implicit teaching potential of a sample of 1.952 activities taken from 10 primary school EFL textbooks used in Spain. Results revealed that the proportion of form-focused activities is higher than expected in the context of EFL textbooks for primary school teaching.

KEYWORDS: Primary School, English as a Foreign Language, explicit and implicit teaching potential, corpus of activities.

\title{
LES ACTIVITÉS D'APPRENTISSAGE DE L'ANGLAIS LANGUE ÉTRANGÈRE DANS LES MANUELS SCOLAIRES POUR L'ÉCOLE PRIMAIRE
}

\begin{abstract}
RÉSUMÉ: Atteindre l'aisance nécessaire dans les compétences orales et écrites est l'un des objectifs les plus importants pour les apprenants d'anglais langue étrangère (ALE). Les différentes activités présentées dans les manuels scolaires peuvent avoir des potentiels différents pour l'enseignement explicite et implicite selon le type de connaissances qu'elles encouragent. Par conséquent, le type de connaissances que les apprenants acquièrent dépendra, dans une large mesure, de l'accent mis par les enseignants sur les différentes activités. Étant donné que les jeunes apprenants tirent davantage profit des activités de communication, cette étude exploratoire vise à examiner le potentiel explicite et implicite d'un échantillon de 1952 activités tirées de 10 manuels scolaires du primaire pour l'enseignement de l'anglais langue étrangère en Espagne. Les résultats ont montré que la proportion d'activités consacrées à l'enseignement des formes dépasse largement ce à quoi on pouvait s'attendre.

MOTS CLÉS: École primaire, anglais langue étrangère, potentiel pédagogique explicite et implicite, corpus d'activités.
\end{abstract}

Recibido: 06/07/2018. Aceptado: 15/11/2018

\section{El potencial de aprendizaje de las actividades para el ILE}

Las actividades para el aprendizaje del ILE son una herramienta fundamental para los profesores (p. ej., Sánchez 2009; Montijano-Cabrera 2014), especialmente 
en contextos donde la exposición al input es pobre en cuanto a cantidad y calidad. Un ejemplo de ello podría ser el aula de lengua extranjera. Dentro de un ámbito académico, la mayoría de actividades suelen promover un tipo de enseñanza centrada en el aprendizaje de formas del lenguaje (Ellis, Basturkmen y Loewen 2002). Para enseñar dichas formas, los profesores pueden emplear diversas actividades que presentarán el contenido -principalmente- de tres maneras: explícita, implícita o mediante una combinación de ambas. Entre las actividades que fomentan el aprendizaje de las formas explícitamente, podemos encontrar ejercicios de (a) revisión y corrección gramatical y ortográfica, (b) respuesta múltiple, (c) unión de formas, (d) compleción de huecos y tablas, (e) pronunciación, (f) búsqueda de vocabulario y formas específicos, (g) reflexión metalingüística, (h) elaboración de listas de vocabulario o reformulación (Gris-Roca 2015: 171). Por otro lado, algunos ejemplos de actividades centradas en el aprendizaje implícito de formas podrían ser los ejercicios de comprensión y producción oral y escrita, además de actividades de (a) respuesta abierta o cerrada, (b) descripción fotográfica, (c) juego de roles, (d) conversación libre o debate argumentado, (e) encuestas o test comunicativos, (f) interacción grupal, (g) búsqueda de información específica, (h) verdadero o falso (Gris-Roca 2015: 172). Finalmente, hay actividades en los libros de texto que promueven - con un cierto grado de equilibrio- la enseñanza de las formas de manera mixta, es decir, utilizando estrategias pedagógicas de carácter explícito e implícito. Entre este último tipo de actividades podríamos encontrar ejercicios que aúnen atención a la forma (p. ej., aprender vocabulario específico relativo a una temática concreta) y atención a la práctica comunicativa y/o pragmática del lenguaje (p. ej., mantener un debate con el profesor o los compañeros acerca de un tema).

Dentro del contexto de la enseñanza del ILE en primaria, hay un aspecto clave que ha de tenerse en cuenta: el estadio de desarrollo del aprendiz. Según la edad y el desarrollo cognitivo de los estudiantes más jóvenes, puede existir una serie de limitaciones específicas asociadas a la capacidad atencional y de procesamiento consciente del conocimiento basado en reglas (p. ej., Baars 1997; Philp, Oliver y Mackey 2008; Paradis 2009; Ullman 2016). Es por ello que los aprendices de ILE -durante su etapa en la escuela primaria- probablemente se beneficien más de aquellas acciones pedagógicas y actividades de aprendizaje de carácter implícito que de materiales docentes y estrategias con un alto potencial de enseñanza explícito. No obstante, si bien es cierto que existe la creencia generalizada de que las intervenciones docentes explícitas para el aprendizaje de las formas de la lengua extranjera pueden ser menos beneficiosas que las acciones implícitas, aún impera la necesidad de examinar empíricamente la correlación entre el uso de ciertas actividades y las ganancias en el conocimiento derivadas de las mismas (Goorhuis- 
Brouwer y de Bot 2010; Azkarai y Imaz-Aguirre 2016; Azkarai y Oliver 2016). Hasta el presente, no parece haberse prestado suficiente atención a ciertas áreas del ILE como: (i) el aprendizaje temprano en el contexto de la escuela primaria (LázaroIbarrola y Azpilicueta-Martínez 2014), (ii) el método de enseñanza y su impacto en el aprendizaje entre aprendices jóvenes (Goorhuis-Brouwer y de Bot 2010), (iii) los efectos de la repetición de tareas (Azkarai y Oliver 2016) y el uso de estrategias de negociación del significado (Azkarai y Imaz-Aguirre 2016) dentro del mismo ámbito. Sin embargo, mucha menos atención se ha prestado a la investigación sobre las tipologías de actividades empleadas en la enseñanza del ILE en el contexto de la escuela primaria o sobre su impacto en el desarrollo cognitivo de los aprendices.

Partir de la premisa de que los niños probablemente se beneficien más de la enseñanza implícita ha llevado algunos autores (p. ej., Ebbels 2007) a creer que existe «un movimiento actual para reducir la intervención directa [o explícita] entre los niños en edad escolar» (p. 67), al menos, entre profesores a cargo de estudiantes jóvenes con trastorno específico del lenguaje (TEL). Sin embargo, no solo son estos aprendices con TEL quienes se exponen con más frecuencia a acciones pedagógicas y materiales docentes de carácter más implícito. Los niños con un desarrollo normal puede que también reciban más instrucción en forma de explicaciones y materiales didácticos implícitos que explícitos, pues tanto a unos aprendices como a otros les caracterizan una serie de rasgos en común, a saber, (a) una menor consciencia de aspectos metalingüísticos y sociolingüísticos, (b) un menor desarrollo de experiencias y habilidades cognitivas y memorísticas y (c) cambios potenciales durante al menos tres etapas distinguibles en la niñez, como la infancia temprana (2-7 años), infancia mediana (7-11 años) y adolescencia temprana (12-14 años) (Lázaro-Ibarrola y Azpilicueta-Martínez 2014: 3-4).

En la literatura sobre la enseñanza del ILE en etapas tempranas, han sido escasos los enfoques para averiguar las preferencias de los aprendices en cuanto a estrategias pedagógicas y materiales docentes, con el fin de mejorar su capacidad de aprendizaje y memorización, la exposición a estímulos lingüísticos y, sobre todo, el ambiente del aula (p. ej., Alizadeh 2011). De manera similar, más investigación es necesaria para examinar los efectos sobre el aprendizaje de determinadas acciones docentes, como, por ejemplo, el uso de la L1 en la clase de ILE. El objetivo de dichas investigaciones debería estar centrado en comprobar si, con el uso de la L1, (i) existe un impacto positivo sobre el desarrollo de la lengua extranjera, (ii) se facilita la retención del conocimiento sobre las formas de la lengua extranjera, (iii) mejora la comprensión en general, como destreza y (iv) se puede llegar a construir un entorno más propicio y efectivo para el 
aprendizaje de la lengua extranjera (Kang 2008: 215). Algunos estudios sobre el bilingüismo (p. ej., Goorhuis-Brouwer y de Bot 2010) han contribuido al desarrollo de la investigación en este campo. Entre los hallazgos encontrados en dicha área, se comenta un escenario en el que los aprendices jóvenes de ILE tienden a mostrar destrezas cognitivas y metacognitivas más avanzadas que los niños monolingües. Entre las destrezas en las que los niños bilingües parecen ser más competentes están, por ejemplo, «las habilidades numéricas, el control sobre la atención y el pensamiento creativo» (Bialystok 2001, 2007; Bialystok, Martin, y Viswanathan 2005; citado en Goorhuis-Brouwer y de Bot 2010: 291).

Otras ramas de la investigación en el campo del aprendizaje temprano del ILE han sido el estudio de la adquisición del vocabulario (p. ej., Agustín-Llach 2015) y, aunque mucho más escaso, el papel de los materiales didácticos en el aula (p. ej., Montijano-Cabrera 2014; Gris-Roca 2017). En última instancia, diversos autores (Ebbels 2007; Goorhuis-Brouwer y de Bot 2010; Lázaro-Ibarrola y Azpilicueta-Martínez 2014; Azkarai y Imaz-Aguirre 2016) han enfatizado su preocupación ante la escasez de estudios empíricos acerca de los posibles efectos derivados de la implementación de distintos métodos y técnicas para la enseñanza del ILE entre aprendices de primaria, teniendo en cuenta $-\mathrm{y}$ prestando especial atención a- la edad y nivel de dominio de la lengua extranjera de los informantes.

\section{Estado de la cuestión}

La enseñanza reglada del Inglés como Lengua Extranjera en la Educación Primaria en España está enmarcada dentro de las especificaciones del Marco Común de Referencia para las Lenguas para cada uno de los cursos que la componen. El planteamiento, los contenidos y la metodología docente que siguen los materiales para la enseñanza del ILE están estipulados en el currículo académico (RDL 126/2014, de 28 de febrero: 40-45) y los libros de texto empleados tienden a seguir las directrices del Método Comunicativo para la enseñanza de lengua extranjera. Los principios cognitivos sobre los que se apoya la secuenciación de las actividades en los libros de texto pertenecientes a la corriente del Método Comunicativo tendrían cabida dentro del contexto de la Teoría de Adquisición de Destrezas (DeKeyser 2015), cuyo fin último es la consecución de fluidez en el manejo de una destreza mediante la automatización del conocimiento. Esta teoría propone que, al igual que para alcanzar el dominio de cualquier destreza, existen tres grandes estadios dentro del proceso de aprendizaje de un idioma extranjero. Estos tres grandes estadios se pueden asociar al modelo clásico de secuenciación 
de actividades: «presentación, práctica y producción» (PPP) (cf. Sánchez 2009; Criado y Sánchez 2009; Criado 2010; Richards y Rodgers 2014).

La primera etapa $(\mathrm{P} 1)$ se centra en mostrar a los alumnos parte del conocimiento declarativo sobre lenguaje (dentro de la Enseñanza Primaria el énfasis recaería sobre el vocabulario y en los tiempos verbales básicos). Los ejercicios característicos al inicio de la unidad en un libro de texto basado en el Método Comunicativo suelen tratar la presentación de vocabulario acerca de una temática y estructuras lingüísticas y tablas para la reflexión metalingüística o compleción de huecos, entre otros (p. ej., Read 2007). Todos ellos constituyen actividades altamente controladas donde la producción libre de lenguaje por parte del alumno es nula o muy reducida.

La segunda etapa (P2) se centraría poner en práctica el conocimiento declarativo (un proceso conocido como «procedimentalización»), aprendido previamente a través de ejercicios que promueven una práctica aún bastante controlada del lenguaje, es decir, actividades en las cuales el alumno tiene todavía una escasa maniobrabilidad en el manejo de la lengua (p. ej., compleción de huecos, reordenación o unión de palabras y frases, lectura y reflexión metalingüística, reformulación, entre otros; ver, por ejemplo, Read 2007).

Por último, en la tercera etapa de producción (P3), se promovería la automatización del conocimiento con el fin de que el alumno agilice los procesos cognitivos de acceso y recuperación de conocimiento progresivamente, sin la necesidad de recurrir a las estructuras y vocabulario (conocimiento declarativo) de forma plenamente deliberada y consciente.

Si bien es cierto que el «qué» (entiéndase como contenido didáctico) y el «cómo» (propuestas pedagógicas, es decir, actividades) de la enseñanza del ILE en la Escuela Primaria están bien enmarcados dentro de los parámetros mencionados, para convenir argumentos objetivos acerca del estado de la investigación en ILE, habría que recordar ciertos apuntes metodológicos. Desde el punto de vista de la concepción de un método de enseñanza, es necesario contar con tres ejes principales (Sánchez 2009). El eje primero serían las creencias y principios sobre los que se sustenta cualquier método. Por ejemplo, dentro del contexto del Método Comunicativo, el principal objetivo del aprendizaje de un idioma extranjero sería la obtención de fluidez en la comunicación. El segundo eje trataría los contenidos concretos que se transfieren al alumno, teniendo en cuenta los principios sobre los que se sostiene dicho método. Y finalmente, el tercer eje convendría la manera en que dichos contenidos se materializan en forma de actividades para 
que el alumno lleve a cabo la práctica lingüística. Atendiendo a este criterio, una gran parte de las publicaciones en el área de la investigación sobre el ILE en la Enseñanza primaria se centran en los ejes 1 y 3 (Halliwell 2000; Brewster, Ellis y Girard 2002; Hearn, Izabella y Garcés-Rodríguez 2003; Pérez y Roig 2004; Read 2007; House 2011). En otras palabras, una gran cantidad de autores se centran en las características del aprendizaje en las edades que comprenden los alumnos de la escuela primaria y ponen de relieve propuestas didácticas que satisfagan las necesidades de dichos estudiantes. En menor medida, se encuentran publicaciones que aborden los contenidos didácticos en el currículo académico desde una postura crítica. En otras palabras, los contenidos curriculares en el área de la enseñanza del ILE en la Escuela Primaria están claramente definidos (RDL 126/2014, de 28 de febrero: 40-45), pero existe menos literatura que presente argumentos para discutir sobre en qué medida dichos contenidos se adecúan a la etapa de aprendizaje desde un punto de vista cognitivo.

\subsection{Fundamentos cognitivos para el aprendizaje del ILE}

Enmarcado en la investigación sobre la Adquisición de Segundas Lenguas (ASL), uno de los objetivos fundamentales de la enseñanza del ILE probablemente sea el logro -por parte de los estudiantes y a través de las acciones y materiales docentes- de conocimiento implícito sobre la lengua extranjera. El objetivo último es que los alumnos puedan alcanzar la fluidez deseada en las destrezas de producción y comprensión (DeKeyser 2015). Para lograr dicha meta, se estima que la enseñanza explícita e implícita pueden ayudar al desarrollo de la L2, pues ambas ejercen un impacto sobre el tipo de conocimiento adquirido por el aprendiz. En la literatura (Hulstijn 2002, 2005), se observa con frecuencia que la enseñanza explícita está estrechamente ligada con el desarrollo de conocimiento explícito del lenguaje. Por otro lado, la enseñanza implícita se asocia más bien con la obtención de conocimiento implícito. Sin embargo, para salvaguardar la precisión y consistencia terminológicas, es necesario aclarar que dichas asociaciones pueden llegar a ser imprecisas. En el contexto del presente estudio se hablará de «Instrucción basada en las formas», pues ésta cubre todas las acciones pedagógicas, tanto explícitas como implícitas (Ellis, Basturkmen y Loewen 2002), cuyo resultado sea el aprendizaje explícito y/o implícito de conocimiento declarativo y/o procedimental de la lengua (Paradis 2009; Ellis 2015; Rebuschat 2015).

Lo que parece cierto es que los acercamientos explícitos a la enseñanza del ILE se caracterizan por (a) el uso de la L2 como principal objeto de estudio, (b) 
la impartición frecuente de explicaciones metalingüísticas durante la lección, (c) la presentación explícita de materiales didácticos y (d) la práctica controlada de las formas. Por otro lado, los enfoques implícitos tienen como característica principal el uso de la L2 como medio de comunicación. Además, se evitan las explicaciones metalingüísticas y se favorece el uso menos controlado de las formas. En ambos casos la acción pedagógica queda operacionalizada con el uso de actividades (Sánchez 2009). Todas las actividades para la enseñanza y el aprendizaje del ILE están diseñadas desde el punto de vista de la enseñanza explícita y/o implícita (Criado, Sánchez y Cantos 2010; Gris-Roca 2015, 2017). Despendiendo del enfoque de cada una, el potencial de enseñanza que les caracteriza también podrá ser mayormente explícito, mayormente implícito o mixto. Este último caso lo representan aquellas actividades que incitan al alumno tanto a reflexionar conscientemente acerca de las formas y posteriormente a usarlas en un contexto comunicativo. A continuación, se mostrarán ejemplos de actividades idiosincráticas que podrían incluirse en esas tres categorías.

En primer lugar, aquellas actividades donde predomina la atención a determinadas formas del lenguaje, y en las que el papel del significado comunicativo o pragmático queda relegado a un segundo plano, tienden a reflejar un mayor potencial de enseñanza explícito. La siguiente actividad podría ser representativa de la enseñanza mayormente centrada en las formas, puesto que su objetivo principal es el aprendizaje de una lista de verbos regulares e irregulares.

\begin{tabular}{|c|c|c|c|c|c|}
\hline \multicolumn{2}{|c|}{ (1) Complete the table. } & \multicolumn{3}{|c|}{$\begin{array}{l}\text { went' ate Ived fgave washed sam played } \\
\text { wrote bought used meade perd drank-tovit }\end{array}$} & $\begin{array}{l}\text { Remember! } \\
\text { Regudar werts end in -ed. }\end{array}$ \\
\hline Infinitive & Past Simple & Infinitive & Past Simple & Infinitive & Past Simple \\
\hline buy & bought & have & had & see & Sow \\
\hline eat & ate & live & lived & use & used \\
\hline drink & drank & love & Poved & wosh & woshed \\
\hline give & gove & make & made & write & wrote \\
\hline go & went & play & played & & \\
\hline
\end{tabular}

Figura 1. Ejemplo de actividad totalmente enfocada a la enseñanza de las formas, con un mayor potencial de enseñanza explícito (Phillips y Shipton 2014: 33).

En segundo lugar, aquellas actividades cuyo objetivo principal es la atención al mensaje comunicativo y donde la atención a las formas es secundaria suelen caracterizarse por un potencial de enseñanza implícito más alto. La actividad en la Figura 2 es un ejemplo de lo que podría considerarse una actividad enfocada 
al significado. Su objetivo principal es entender el mensaje en la grabación para numerar correctamente las figuras.

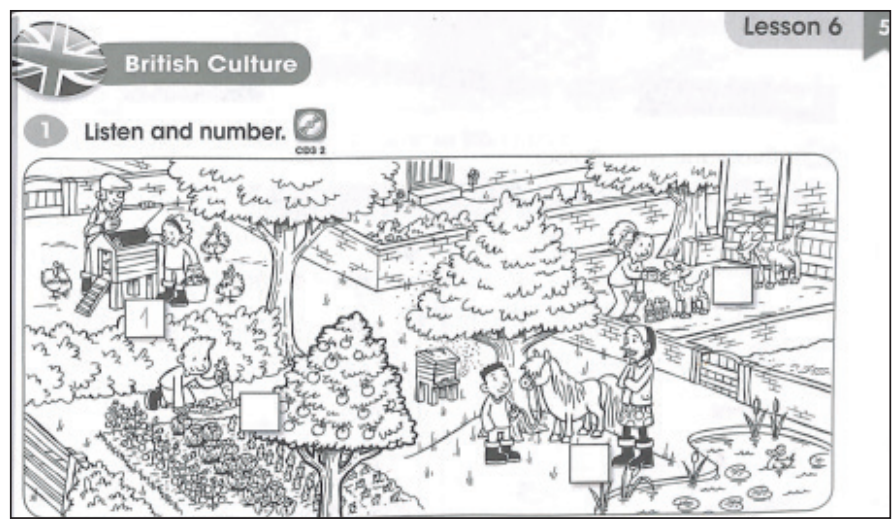

Figura 2. Ejemplo de una actividad mayormente enfocada a la enseñanza del significado comunicativo y/o pragmático, con un potencial de enseñanza implícita más alto (Shawn y Ramsden 2014: 53).

En tercer lugar, las actividades que demandan que el alumno preste atención tanto a la forma como al contenido suelen tener un potencial de enseñanza mixto, es decir, parcialmente explícito e implícito. Por ejemplo, la actividad de la Figura 3 requiere atención a la forma -más específicamente, a la conjugación de verbos en pasado simple, continuo y perfecto- y al contenido del mensaje comunicativo y/o pragmático. Si bien es cierto que la actividad exige un tipo de práctica bastante controlada (p. ej., elegir la forma adecuada del verbo), involucrar al alumno en el procesamiento del input mediante la práctica receptiva podría conducir a la automatización del conocimiento. Esto, en cambio, promueve la enseñanza y el aprendizaje implícitos.

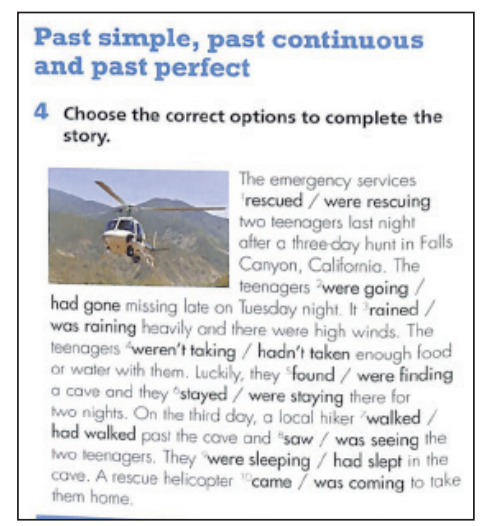

Figura 3. Ejemplo de una actividad parcialmente enfocada en la enseñanza de las formas y el significado, con un potencial de enseñanza mixto (Goldstein y Jones 2015: 22). 
Las actividades de libros de texto empleadas para enseñar a aprendices jóvenes comparten una serie de propiedades (cf. Scott y Ytreberg 1990; Halliwell 2000; Brewster, Ellis y Girard 2002; Hearn, Izabella y Garcés-Rodríguez 2003; Pérez y Roig 2004; Read 2007). Más concretamente, se podría hacer una distinción entre las actividades que se implementan con grupos de estudiantes de entre 5 a 7 años de edad y las que se usan con estudiantes de entre 8 y 10 años. Siguiendo la distinción que Scott e Ytreberg (1990) establecen, se mostrarán a continuación algunas de las características fundamentales que deberían destacar en las actividades para la enseñanza del ILE en la educación primaria.

Las actividades diseñadas para niños de entre cinco y siete años comparten las siguientes propiedades:

- ignoran la información y reglas metalingüísticas explícitas, pues los estudiantes más jóvenes puede que aún no posean las destrezas cognitivas suficientes para entenderlas, interpretarlas o procesarlas de manera consciente;

- requieren el uso de las cuatro destrezas básicas, a saber, la escritura, el habla, la escucha y la lectura;

- son variadas y atraen la atención hacia distintos aspectos entre las distintas tareas, con el fin de mantener a los aprendices concentrados;

- divierten, retan y recompensan al estudiante. Tanto los juegos como los retos pueden ser motivadores y hacer que se sientan valorados por su esfuerzo, trabajo y logros;

- favorecen el aprendizaje colaborativo y la competición;

- recogen los intereses y las inquietudes de los aprendices, preguntándoles por lo que les gusta, lo que están haciendo o sus experiencias;

- ayudan al desarrollo de destrezas cognitivas importantes a través del razonamiento lógico y la imaginación;

- usan elementos visuales y lingüísticos (p. ej., narrativas, ilustraciones);

- facilitan la interacción entre el profesor y el alumno para comprobar el nivel de comprensión de este último. En algunas ocasiones, los aprendices jóvenes suelen permanecer en silencio o fingir que entienden los contenidos antes que admitir que no saben algo.

Por otro lado, las actividades diseñadas para niños de entre ocho y diez años se caracterizan por:

- introducir conceptos metalingüísticos básicos y explicaciones breves acerca de las formas para facilitar la inferencia de los rasgos lingüísticos menos destacados en el input; 
- mostrar información extra sobre la L2 para promover el interés de los estudiantes más avanzados;

- animar a los aprendices a formular preguntas en la L2;

- emplear un número moderado de pistas visuales (p. ej., fotografías, dibujos) y auditivas (p. ej., entonación, acentuación) para transmitir el significado de los textos y grabaciones;

- crear conciencia acerca de las formas de la L2 mediante la manipulación del lenguaje con juegos;

- promover el aprendizaje cooperativo y la competición entre alumnos;

- ser más variadas en términos de tipología de actividades, dado que a mayor madurez los alumnos puede que hayan desarrollado una actitud más crítica acerca del tipo de actividades que prefieren o detestan hacer.

\subsection{La importancia de mantener el interés de los alumnos}

Tras enumerar las características idiosincráticas de las actividades para jóvenes aprendices del ILE, es necesario enfatizar el papel de un factor clave: la atención. Para que el aprendizaje tenga lugar tanto dentro como fuera del aula $-\mathrm{y}$ como parte fundamental dentro del constructo de aprendizaje propuesto por Leow (2015)- los estudiantes deben prestar atención. Probablemente solo de esta manera, parte del input pueda ser procesado e interiorizado, para posteriormente ser almacenado en la memoria como pa+rte del conocimiento adquirido de la L2. Por lo tanto, el conseguir que los estudiantes más pequeños presten atención a formas específicas del lenguaje y a su significado a través de las actividades podría constituir una de las tareas más complejas para el profesorado del ILE (p. ej., Alcón-Soler y GarcíaMayo 2008). Una serie de investigadores en el área (p. ej., Scott y Ytreberg 1990; Shin 2006; Philp et al. 2008) han subrayado la importancia no solamente del input lingüístico, sino también de los elementos visuales, entre otros aspectos. Éstos pueden ser enfatizados a través de la utilización de una variedad representativa de estímulos en el aula. Los autores mencionados nombran una serie de estrategias para atraer la atención que parecen compatibles con el uso equilibrado de las distintas tipologías de actividades en los libros de primaria para la enseñanza del ILE.

\subsection{Posibles vías de estímulo para captar la atención de los alumnos}

La creencia común acerca del grado de control consciente que los niños pueden llegar a ejercer sobre sus recursos atencionales ha derivado en numerosas opiniones. 
La capacidad más reducida en el control de los periodos de atención en los niños (en comparación con los adultos) se podría contrarrestar con el comportamiento energético y la predisposición que demuestran a la hora de descubrir y aprender nuevos conocimientos (Philp et al. 2008). De manera similar, Scott e Ytreberg (1990) y Shin (2006: 5) señalan que «las palabras no son suficientes». La combinación de los elementos visuales, los objetos, los juguetes, las marionetas - o lo que en inglés se conoce como «realia», tan característico del acercamiento natural, una corriente previa al Método Directo, en la enseñanza de idiomas extranjeros (Sánchez 2009)-, los movimientos corporales y los gestos (p. ej., como en la Respuesta Física Total) es fundamental. Todo ello puede ser beneficioso cuando se incorpora al uso de actividades en el aula. Además, la inclusión de actividades prototípicas de otras materias o disciplinas, como las asignaturas de ciencias, podría impulsar la atención de los aprendices. Un ejemplo de ello serían las manualidades (p. ej., Wright 2008). No obstante, determinadas actividades más características de las áreas de educación física, música y tecnología, donde la interacción y la exposición al input lingüístico son menos frecuentes, deberían implementarse con moderación (Van den Branden 2008: 156). Cuantas más restricciones haya en el plano de la exposición al input y en la interacción verbal en la lengua extranjera, el número de oportunidades para el aprendizaje podría disminuir.

\subsection{El uso de distintos materiales docentes cómo estímulo atencional}

Mantener a los aprendices concentrados a lo largo de toda la lección podría ser más sencillo con el empleo de actividades variadas. Pasar de una actividad a otra, dedicándole no más de diez o quince minutos a cada una podría mejorar la capacidad de los alumnos para prestar atención. En este sentido, Scott e Ytreberg (1990: 102) aconsejan la implementación de actividades de tipologías distintas de una manera consecutiva. Por ejemplo, (a) actividades para comunicarse oralmente antes o después de otras en las que haya que trabajar en silencio y de manera individual, (b) actividades en grupo antes o después de otras en las que se pregunte a toda la clase, (c) actividades que fomenten la interacción alumno con alumno frente a otras en las que sea el profesor quien interactúe con el alumno, o (d) actividades en las que se hagan juegos como medio para aprender el lenguaje frente a otras en las que toda la atención se focalice en la manipulación consciente de las formas del lenguaje.

\subsection{El fomento de una actitud participativa}

Con el objetivo de suscitar la atención y el interés de los alumnos acerca del mundo que les rodea, los materiales deberían transmitir el conocimiento 
de la L2 a través de diversos temas. Algunos de los temas más comunes que se emplean para enseñar a aprendices jóvenes son «los animales, los amigos y la familia, o las unidades que traten los libros de cuentos» (Shin 2006: 4). A lo largo del desarrollo cognitivo de los estudiantes, en el curriculum escolar van apareciendo otros temas con un grado mayor de complejidad, como, por ejemplo, el medio ambiente o la ciudadanía. Lo que parece cierto es que enseñar el ILE empleando temas que puedan resultar familiares para los alumnos podría tener beneficios en la adquisición del lenguaje (Philp et al. 2008). No obstante, para hacer un uso adecuado de los temas en este contexto, hay tres condiciones indispensables: que estén adaptados al nivel de dominio de los estudiantes, que se haga un uso coherente de los mismos durante la lección y que cumplan con los objetivos de aprendizaje en el currículum. Además, el contenido de materias similares podría ofrecer oportunidades tanto para el profesor como para los alumnos para aprender o «reciclar» el conocimiento previo (p. ej., vocabulario visto en unidades anteriores).

\subsection{El uso de rutinas como estímulo de aprendizaje}

La previsibilidad en cuanto al uso de materiales docentes o la actuación del profesor en el aula podría favorecer un entorno positivo y cómodo para los niños, pudiendo reducir así su nivel de estrés y aumentando la concentración. Para el profesor del ILE, seguir «rutinas [específicas] en el aula puede ayudar a controlar a los alumnos» (Shin 2006: 5). Las rutinas de clase ocurren cada día y pueden aportar oportunidades para desarrollar la lengua extranjera (Cameron 2001), siempre y cuando el profesor implemente -o diseñe- actividades que exijan interacción. Entre las estrategias para atraer la atención de los alumnos, como es el empleo de nombres o «títulos» para las distintas secciones de la lección (p. ej., «The Friday Quiz»), pueden resultar de utilidad para crear hábitos y rutinas cada semana (Cekaite 2008).

\subsection{Las ventajas en el empleo de la L1 en el aula}

Los intentos por reducir las intervenciones del profesor de ILE en la lengua materna de los estudiantes puede que no sea siempre favorable para todos ellos (e.g. White 2008). La L1 puede ser un instrumento docente muy útil, especialmente cuando se intenta transmitir el significado de palabras o expresiones más complejas. También, el empleo de la L1 puede favorecer la motivación entre aquellos que encuentren difícil seguir el ritmo de la clase si 
solo se emplea la lengua extranjera. Usar la L1 en determinadas circunstancias puede ayudar al profesor a gestionar y ahorrar tiempo con las explicaciones y el resultado en términos de aprendizaje de las mismas (Shin 2006). Esto, sin embargo, no debería implicar que se remplace la lengua extranjera por la materna durante toda la lección.

\section{Objetivo}

El objetivo de este estudio exploratorio fue examinar la naturaleza pedagógica (explícita, implícita o mixta) de 1.952 actividades extraídas de 10 libros frecuentemente usados para la enseñanza del ILE en la escuela primaria en España -a mi saber, hasta la fecha solo existe un estudio previo (Gris-Roca, 2017) realizado con una muestra de solo 100 actividades. El objetivo final fue verificar si las actividades en sí mismas están diseñadas teniendo en cuenta las limitaciones potenciales en la enseñanza de una lengua extranjera entre aprendices de primaria.

\section{Método}

Para comprobar el potencial de enseñanza de las 1.952 actividades, una serie de subtareas fueron llevadas a cabo. La primera de ellas implicaba la recopilación de una muestra de actividades de libros de texto para la enseñanza del ILE de entre una variedad de libros cuyo uso está extendido en el ámbito de la escuela primaria en España (vea las secciones 4.1 y 4.1.1). La segunda subtarea consistía en trasvasar todas las instrucciones de las actividades (es decir, el encabezamiento $\mathrm{o}$ «heading») de la muestra de 10 libros de texto a un documento Excel (vea la sección 4.1.2). La tercera subtarea implicaba la aplicación de un instrumento de medida para verificar el potencial pedagógico explícito y/o implícito de las actividades (vea la sección 4.2). Por último, la cuarta subtarea tenía como objetivo la validación del proceso de categorización de las actividades mediante el análisis del grado de fiabilidad interjuez. A continuación, se describirá cada uno de los pasos en detalle.

\subsection{Materiales para el análisis}

Un conjunto de 36 libros de texto populares dentro del ámbito de la enseñanza del ILE en España (vea el Apéndice 1) fueron usados para compilar 
la muestra de actividades. Se tuvo en cuenta tanto el libro del alumno como el cuaderno a la hora de hacer la selección aleatoria. Debe advertirse, sin embargo, que bajo ningún concepto se incurrió en una infracción de los derechos de autor, pues el análisis de los libros de texto y sus actividades se llevó a cabo con fines estrictos de investigación. Los materiales proceden de algunas de las editoriales más conocidas en el área a nivel nacional, a saber, Oxford University Press, Cambridge University Press, Macmillan, Pearson Longman, y Richmond Publishing.

\subsubsection{Muestra de libros de texto}

De todo el conjunto de 36 libros de texto de primaria para la enseñanza del ILE, se hizo una selección aleatoria de una muestra de 10 libros de nivel básico (A1, A2), todos ellos publicados entre 2004 y 2015, e implementados en el segundo ciclo - comprendido entre tercero y cuarto de primaria- y en el tercer ciclo, que abarca los cursos de quinto y sexto. Uno de los criterios para garantizar la aleatoriedad del proceso de selección de libros de texto para la muestra fue que éstos pertenecieran a diferentes casas editoriales. Tras el proceso de selección, 5 libros pertenecían a la editorial Oxford University Press, 2 a Macmillan, otros 2 a Longman y, finalmente, 1 a Richmond. Todas las actividades (incluidas las actividades adicionales en las unidades de revisión) en cada uno de los 10 libros que se muestran abajo (ver la Tabla 1) formaron parte del «corpus» de actividades.

Tabla 1. Muestra de 10 libros de texto para la enseñanza del ILE en la escuela primaria

\begin{tabular}{|l|l|}
\hline Big Surprise 5 (libro del alumno) & Find Out 3 (libro del alumno) \\
\hline Big Surprise 6 (cuaderno) & Find Out 3 (cuaderno) \\
\hline Great Explorers 6 (libro del alumno) & English Adventure 3 (libro del alumno) \\
\hline Great Explorers 6 (cuaderno) & English Adventure 3 (cuaderno) \\
\hline Oxford Rooftops 3 (cuaderno) & Mega Zoom 3 (libro del alumno) \\
\hline
\end{tabular}

4.1.2. Selección, análisis, categorización y codificación de las actividades

Todas las actividades de los 10 libros de texto aleatoriamente seleccionados fueron extraídas, analizadas y categorizadas individualmente. Si bien es cierto que el proceso de análisis y categorización fue individual, se tuvo en 
consideración la secuenciación de las actividades para determinar su potencial de enseñanza. Por ejemplo, casos en los que una actividad de carácter comunicativo se encuentra en la secuencia justo después de otra donde se presenta una forma gramatical y la inclusión de ésta última es necesaria en dicho ejercicio comunicativo.

Para la recopilación de actividades se usó una hoja de Excel y para su categorización se incluyeron una lista de metadatos con el fin de poder localizar la fuente de cada ejercicio en cualquier momento. De izquierda a derecha in la Figura 4 abajo, se incorporan datos relativos al: (i) nombre del libro de texto, (ii) la categoría en cuanto a la edición (SB -del inglés «student's book»-para libros del alumno y WB -del inglés «workbook»-para cuadernos), (iii) la unidad y la página donde se encuentra la actividad, (iv) el número del ejercicio, (v) el tipo de actividad teniendo en cuenta la destreza que promueve, a saber, la producción escrita y oral, y la comprensión lectora y oral, el conocimiento de la gramática, la pronunciación, la ortografía y el vocabulario (se debe tener en cuenta que varias de estas destrezas podían estar

\begin{tabular}{|c|c|c|c|c|c|c|c|}
\hline A & B & c & D & E & $\mathbf{F}$ & G & $\mathrm{H}$ \\
\hline English Adventure (3) & SB & RVW3 & 66 & 2 & L\&S & Listen and answer & 100 \\
\hline English Adventure (3) & SB & RVW3 & 66 & 3 & $\mathbf{R}$ & Read and find & 100 \\
\hline English Adventure (3) & SB & RVW3 & 67 & 4 & S & Look and say & 100 \\
\hline English Adventure (3) & SB & RVW3 & 67 & 5 & MX & Make a scary card & 50 \\
\hline English Adventure (3) & SB & RVW3 & 68 & 1 & L\&S & Listen and find. Then say & 100 \\
\hline English Adventure (3) & SB & RVW3 & 68 & 2 & S & Chant & 100 \\
\hline English Adventure (3) & SB & RVW3 & 68 & 3 & L\&S & Listen and say: yes or no & 100 \\
\hline English Adventure (3) & SB & RVW3 & 69 & 4 & $\mathbf{s}$ & Look. What can you see? & 100 \\
\hline English Adventure (3) & SB & RVW3 & 69 & 5 & R\&S & Read and play & 100 \\
\hline English Adventure (3) & SB & RVW3 & 69 & 6 & MX & Make a christmas tree & 50 \\
\hline English Adventure (3) & SB & RVW3 & 70 & 1 & L\&S & Listen and find. Then say & 100 \\
\hline English Adventure (3) & SB & RVW3 & 70 & 2 & S & Chant & 100 \\
\hline English Adventure (3) & SB & RVW3 & 71 & 3 & S & Look. What can you see? & 100 \\
\hline English Adventure (3) & SB & RVW3 3 & 71 & 4 & $\mathbf{s}$ & Make new words & 100 \\
\hline English Adventure (3) & 58 & RVW3 & 71 & 5 & MX & Make and play & 50 \\
\hline English Adventure (3) & WB & 0 & 2 & 1 & $R$ & Read and match & 100 \\
\hline English Adventure (3) & WB & 0 & 2 & 2 & $\mathrm{v}$ & look and match & 0 \\
\hline English Adventure (3) & WB & 0 & 3 & 3 & L\&W & Listen and write & 100 \\
\hline English Adventure (3) & WB & 0 & 3 & 4 & v & Read and colour & 0 \\
\hline English Adventure (3) & WB & 0 & 4 & 5 & v & Count and write & 0 \\
\hline English Adventure (3) & WB & 0 & 4 & 6 & v & Find & 0 \\
\hline English Adventure (3) & WB & 0 & 5 & 7 & w & Draw and write & 100 \\
\hline English Adventure (3) & WB & 0 & 5 & 8 & v & Find and order & 0 \\
\hline English Adventure (3) & WB & 1 & 6 & 1 & v & Read and colour & 0 \\
\hline English Adventure (3) & WB & 1 & 6 & 2 & R & Look at activity 1 . Read and circle & 100 \\
\hline English Adventure (3) & WB & 1 & 7 & 3 & $v$ & Read and match & 0 \\
\hline English Adventure (3) & WB & 1 & 7 & 4 & L & Listen and show & 100 \\
\hline English Adventure (3) & WB & 1 & 8 & 5 & w & Write & 100 \\
\hline English Adventure (3) & WB & 1 & 8 & 6 & $R \& W$ & Read and write. What is it? & 100 \\
\hline English Adventure (3) & WB & 1 & 9 & 7 & R & Read and circle & 100 \\
\hline English Adventure (3) & WB & 1 & 9 & 8 & $v$ & Draw a toy and write & 0 \\
\hline English Adventure (3) & WB & 1 & 10 & 9 & v & Find and write & 0 \\
\hline English Adventure (3) & WB & 1 & 10 & 10 & v & Draw and write & 0 \\
\hline English Adventure (3) & WB & 1 & 11 & 11 & L & Listen and colour & 100 \\
\hline English Adventure (3) & WB & 1 & 11 & 12 & v & Find and write & 0 \\
\hline English Adventure (3) & WB & 2 & 12 & 1 & L & Listen and colour & 100 \\
\hline English Adventure (3) & WB & 2 & 12 & 2 & $\mathrm{v}$ & Look at activity 1. Read and circle & 0 \\
\hline
\end{tabular}

Figura 4. Captura de pantalla mostrando ejemplos de la categorización de las actividades en Excel. 
presentes a la vez en determinadas actividades), y (v) el encabezamiento de la actividad, es decir, las instrucciones.

Con el fin de codificar las actividades, dos expertos en el área examinaron todas las actividades individualmente. Para ello, se leyeron primero las instrucciones para cada una de las actividades y, seguidamente, se comprobó la secuenciación de las mismas. En tercer lugar, las actividades se categorizaron según su potencial de enseñanza. Tal y como se puede observar en la Figura 4 (ver la columna de la derecha), el código $<0>$ se empleó con aquellas actividades donde la atención del alumno se centraba mayormente en las formas y donde el significado pragmático y/o comunicativo fuera secundario para su compleción. Por otro lado, se asignó el código $<100>$ a aquellas actividades enfocadas en mayor medida al significado, es decir, en las que la atención a las formas del lenguaje constituyera un objetivo secundario. Por último, el código $<50>$ se utilizó con las actividades que requerían atención tanto a las formas como al contenido.

\subsection{Evaluación de la validez en el proceso de análisis}

La validez y fiabilidad interjuez del proceso de análisis, categorización y codificación de las actividades se verificó usando un cálculo estadístico: el Kappa de Cohen, pues en el proceso de validación participaron no más de dos jueces (el Kappa de Fleiss se usa cuando intervienen tres o más jueces en el análisis).

En la Tabla 2 abajo, se entrecruzan los datos resultantes del análisis de los evaluadores para mostrar las coincidencias y desajustes. Tal y como se puede observar, hubo una coincidencia absoluta entre los evaluadores teniendo en cuenta el análisis de las actividades de carácter mayoritariamente explícito $(<0>)$. También hubo 33 de 37 aciertos posibles en la categorización de las actividades cuyo potencial de enseñanza implícito $(<100>)$ era más alto. De forma similar, se obtuvieron 40 aciertos - de los 43 posibles- en la evaluación de las actividades con un potencial pedagógico mixto $(<50>)$. Desde un punto de vista global, de entre 100 aciertos posibles, los evaluadores 1 y 2 consiguieron 93. Esto sugiere que la fiabilidad interjuez era muy alta.

Tras la aplicación del Kappa de Cohen (ver la Tabla 3), se obtuvo el valor exacto para la fiabilidad interjuez $(\mathrm{x}=0,89)$. Esto demuestra el alto grado de validez en la categorización y análisis de las actividades por parte de los evaluadores. 
Tabla 2. Coincidencias entre los evaluadores

\begin{tabular}{|l|c|c|c|c|}
\hline Evaluador 1 & $\mathbf{0}$ & $\mathbf{5 0}$ & $\mathbf{1 0 0}$ & Total \\
\hline 0 & 20 & 2 & 0 & 22 \\
\hline 50 & 0 & 40 & 4 & 44 \\
\hline 100 & 0 & 1 & 33 & 34 \\
\hline Total & 20 & 43 & 37 & 100 \\
\hline
\end{tabular}

Tabla 3. Test de confianza interjuez mediante el coeficiente de Kappa de Cohen

\begin{tabular}{|c|c|c|c|}
\hline \multirow{2}{*}{ Coeficiente } & \multirow{2}{*}{ Valor } & \multicolumn{2}{|c|}{ Intervalo de confianza (9\%) } \\
\cline { 3 - 4 } & & Menor & Mayor \\
\hline Kappa de Cohen & 0,891 & 0,812 & 0,969 \\
\hline
\end{tabular}

\section{Resultados}

En esta sección, se analizarán los resultados desde tres perspectivas distintas. En primer lugar, se expondrá un resumen general de los resultados (vea la sección 5.1) observando la cantidad de actividades por tipología presentes en la muestra. En segundo lugar, se mostrarán los resultados de cada libro de texto ordenados por fecha de publicación con el fin de detectar posibles cambios en la selección de actividades según su tipología en estos últimos años (sección 5.2). En tercer lugar, se buscarán tendencias en los datos mediante la aplicación de un análisis de conglomerado -o clúster (sección 5.3).

\subsection{Resultados generales}

En base a la categorización de todas las actividades de la muestra, se puede observar en la Figura 5 que hay una presencia muy equilibrada tanto de actividades con un potencial de enseñanza explícito más alto $(<0>, \mathrm{n}=721)$ como de ejercicios con una carga implícita mayor $(<100>, \mathrm{n}=713)$. No se puede ignorar, sin embargo, la importante presencia de actividades cuya naturaleza de enseñanza es mixta, es decir, parcialmente explícita e implícita $(<50>, \mathrm{n}=454)$. 
La tendencia en la inclusión de un número equilibrado - casi a la perfecciónde actividades con carácter mayormente explícito y de actividades con un potencial de enseñanza implícito más alto no parece estar en línea con las propuestas pedagógicas expuestas en la literatura acerca de la enseñanza del ILE en el contexto de la Educación Primaria (Scott y Ytreberg 1990; GoorhuisBrouwer y de Bot 2010; Lázaro-Ibarrola y Azpilicueta Martínez 2014). Otorgar dicha importancia al impacto sobre el aprendizaje de las actividades mayormente explícitas puede reflejar que:

- el modo deductivo se podría considerar tan beneficioso como el inductivo para la enseñanza de las formas de la lengua extranjera también en contextos de enseñanza primaria;

- se refuerzan el aprendizaje de componentes específicos como el vocabulario, la gramática, la pronunciación o el deletreo al igual que otras destrezas integrativas como el habla;

- las explicaciones metalingüísticas pueden resultar útiles para los aprendices jóvenes;

- no se ignoran determinadas estrategias (p. ej., aprendizaje deductivo) para estimular la atención de los alumnos.

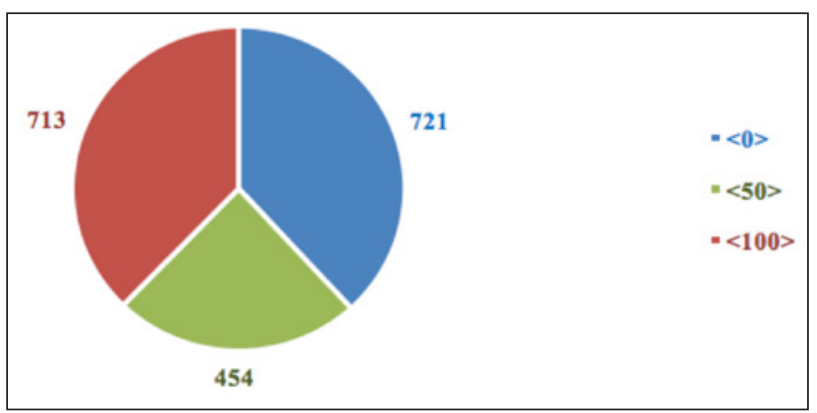

Figura 5. Tipología y cantidad de actividades en la muestra total.

\subsection{Resultados individuales por cada libro de texto}

Atendiendo a los datos mostrados en la Figura 6, se puede observar que han existido fluctuaciones en cuanto a la inclusión de actividades por tipología en los libros de texto. En otras palabras, la disposición de actividades según su tipología en los libros de texto no parece ser predecible. No obstante, este hallazgo sí 
parece estar en consonancia con los cambios en la legislación en el ámbito de la enseñanza de lenguas extranjeras (RDL 126/2014, de 28 de febrero: 38-45) durante esta última década.

Haciendo un análisis más pormenorizado de los datos, en primer lugar, se percibe que los libros de texto con un mayor porcentaje de actividades en la categoría $\langle 100\rangle$, con mayor carga de enseñanza implícita, son English Adventure 3 (libro del estudiante), Find Out 3 (libro del estudiante) y Great Explorers 6 (libro del estudiante). Mientras que los dos primeros se usan en $3^{\circ}$ de primaria (segundo ciclo), donde los alumnos tienen entre 8 y 9 años, el tercero, Great Explorers 6, se imparte en $6^{\circ}$ de primaria (final del tercer ciclo), con alumnos de edades comprendidas entre los 10 y los 11. Teniendo en cuenta la tipología de actividades según su carga de enseñanza explícita, implícita o mixta, se observa que en estos tres libros de texto las actividades enfocadas a la enseñanza de formas tienen una presencia menor, o incluso nula. Aunque se recomienda que las actividades de carácter explícito empiecen a aparecer cuando los estudiantes tienen 8 años (Scott y Ytreberg 1990; Goorhuis-Brouwer y de Bot 2010), algunos libros de texto (p. ej., English Adventure 3 , tanto el libro del alumno como el cuaderno) no parecen incluir ninguna, al menos, exclusivamente enfocada en las formas.

En segundo lugar, entre los libros con más porcentaje de actividades con un potencial mixto $(<50>$ ) se encuentran Mega Zoom 3 (libro del estudiante), Big Surprise 5 (libro del estudiante) y Big Surprise 6 (cuaderno). Mientras que el primero de ellos se utiliza en el segundo ciclo de primaria, tanto el segundo como el tercero pertenecen al tercer ciclo. Llegados a este punto en el análisis, invito a los lectores a reflexionar acerca del alto porcentaje de actividades enfocadas exclusiva o parcialmente a la enseñanza de formas en el contexto de la enseñanza del ILE en la escuela primaria. Este hallazgo contradice los datos recogidos en una muestra menor (Gris-Roca 2017), pues, al no ser estadísticamente representativa, éstos podían proyectar una tendencia muy distinta, más favorable con la dimensión comunicativa del lenguaje. No obstante, los datos del presente estudio sugieren que la selección de actividades para su inclusión en los libros de texto para el ILE no parece ser la más beneficiosa -en términos de cogniciónsegún las directrices encontradas en la literatura.

En tercer lugar, los libros con más porcentaje de actividades con una mayor carga de enseñanza explícita $(<0>)$ son Oxford Rooftops 3 (cuaderno), English Adventure 3 (cuaderno) y Big Surprise 6 (cuaderno). Estos hallazgos también contrastan con los encontrados en Gris-Roca (2017), pues, tras el análisis de una muestra más reducida de actividades, se proyectaba el efecto contrario en el caso 
de muchos de los cuadernos, con mayores cantidades de actividades de naturaleza mixta $(<50>)$ o comunicativa $(<100>)$. En el presente estudio, el porcentaje de actividades mayormente enfocadas a las formas $(<0>)$ siempre supera el $45 \%$ en el caso de los cuadernos de ejercicios. Si bien es cierto que la presencia de actividades para la enseñanza explícita es relativamente alta en los cuadernos, se observa que el porcentaje de actividades para la enseñanza implícita o mixta es más elevado en los libros del estudiante.

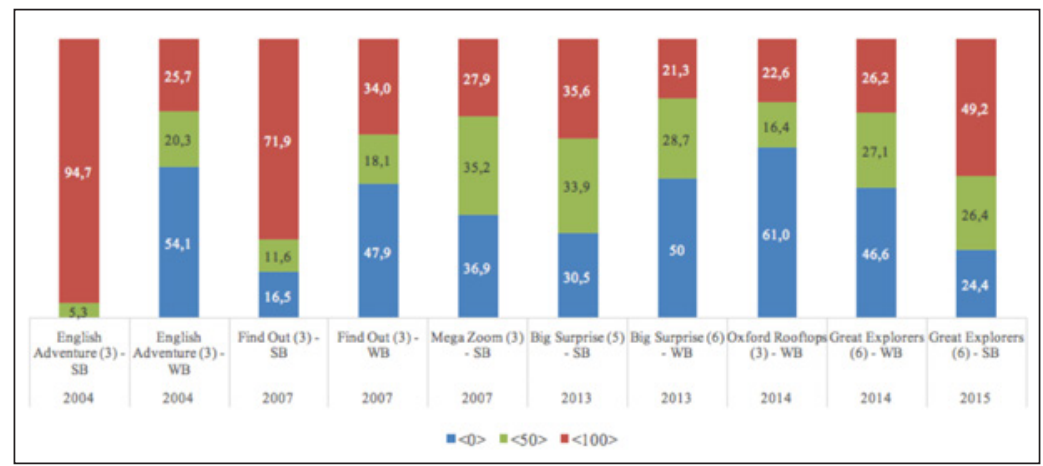

Figura 6. Porcentaje de actividades por tipología y año de publicación en cada uno de los libros de la muestra.

\subsection{Recomendaciones en base a la presencia de actividades}

Con el fin de detectar y verificar estadísticamente similitudes y diferencias entre los libros analizados en cuanto a la cantidad y tipología de actividades, se llevó a cabo un análisis clúster. Este tipo de cálculo puede resultar útil para prever determinadas tendencias en los datos de una manera visual. El resultado de un análisis clúster es un dendograma (ver la Tabla 4), donde se observan ramificaciones que representan tendencias numéricas.

Desde un punto de vista general, se perciben tres grandes ramificaciones. Los casos en la rama del extremo superior -1 y 3 -, que se corresponden con los libros English Adventure 3 (libro del estudiante) y Find Out 3 (libro del estudiante), mantienen una tendencia clara en cuanto a la inclusión de más actividades comunicativas. También reflejan una tendencia similar los casos 6 y 9 en el extremo inferior, correspondientes a los libros Great Explorers 6 (libro del estudiante) y Big Surprise 5 (libro del estudiante) respectivamente. La diferencia entre ambos extremos reside en que, en el inferior, el énfasis comunicativo va 
acompañado de un porcentaje ingente y equilibrado de actividades enfocadas a la enseñanza de las formas y actividades con potencial de enseñanza mixto. En cuanto a la ramificación intermedia, $\operatorname{los}$ casos $8,4,2,5,7$ y 10 , correspondientes a los libros Mega Zoom 3 (libro del estudiante), Find Out 3 (cuaderno), English Adventure 3 (cuaderno), Oxford Rooftops 3 (cuaderno), Great Explorers 6 (cuaderno) y Big Surprise 6 (cuaderno), reflejan una mayor presencia de actividades con un potencial de enseñanza explícito.

Como resultado de esta inspección de los datos, se podría argumentar que los libros más integrativos y adecuados -desde un punto de vista cognitivo y por la elevada carga comunicativa- para la enseñanza del ILE dentro del contexto de la escuela primaria son: English Adventure 3 (libro del estudiante), Find Out 3 (libro del estudiante) y Great Explorers 6 (libro del estudiante).

Tabla 4. Análisis clúster de la muestra completa de actividades

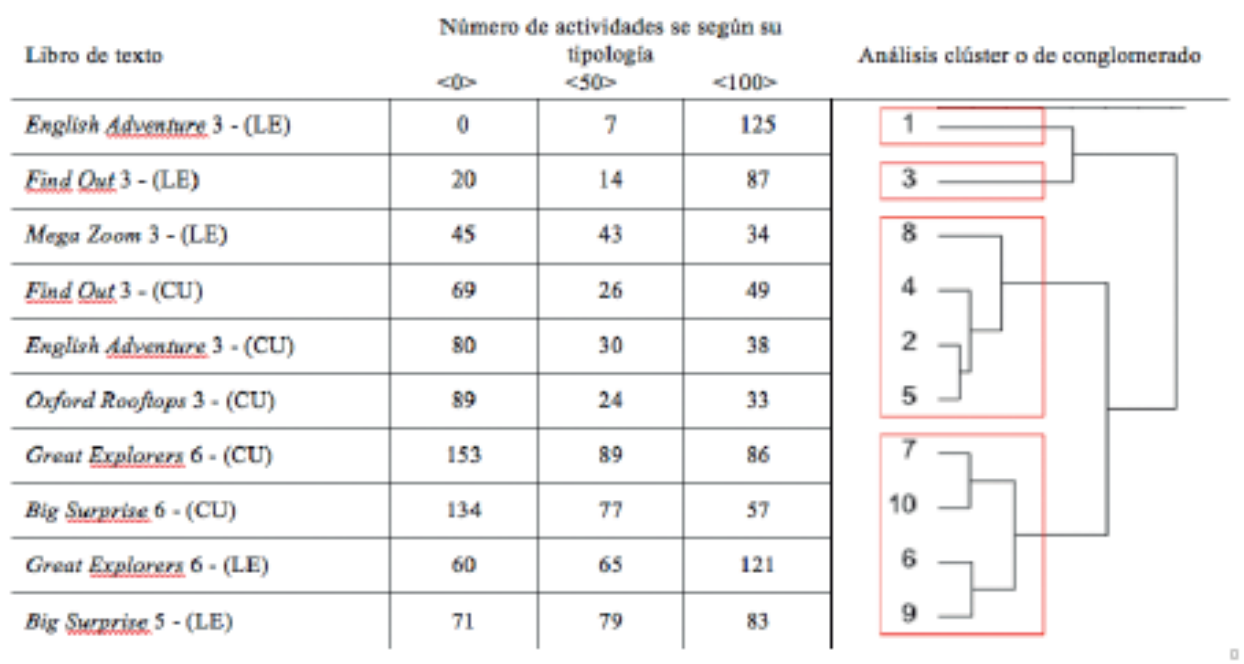

(LE): Libro del estudiante; (CU): Cuaderno

\section{Discusión}

El objetivo del presente estudio exploratorio era descubrir la naturaleza pedagógica de una muestra de actividades para la enseñanza del ILE en la escuela primaria. Publicaciones recientes en el área se han centrado en: (i) la 
interacción entre los aprendices durante la realización de la tarea (cf. LázaroIbarrola y Azpilicueta-Martínez 2014; Azkarai y Oliver 2016), (ii) enfoques sobre la enseñanza del ILE en diferentes contextos de instrucción y con estudiantes con diferentes condiciones (cf. Ebbels 2007; Kang 2008), (iii) la función del libro de texto en el aula de ILE (Carol 2007; Montijano-Cabrera 2014; Gris-Roca 2015, 2017), (iv) el desarrollo del vocabulario entre los aprendices y a través de diversas etapas de la escuela primaria (Barreras 2004; Agustín-Llach 2015) y (v) la actuación pedagógica y sus efectos sobre el dominio de la lengua de los aprendices (Halliwell 2000; Brewster et al. 2002; Hearn y Garcés-Rodríguez 2003; Pérez y Roig 2004; Carol, 2007; Goorhuis-Brouwer y de Bot 2010; House 2011).

Los hallazgos de este trabajo revelaron que -en el caso de los libros de texto para la enseñanza del ILE en la escuela primaria- se tiende a incluir actividades explícitas, implícitas y mixtas, al parecer, sin un criterio selectivo teniendo en cuenta las características básicas del tipo de desarrollo cognitivo de los estudiantes, especialmente, en el estadio de edad que abarcaba la muestra en el presente trabajo (desde tercero a sexto de primaria). Esta falta de criterio en la selección de actividades docentes parece ir en contra de las directrices que determinados autores postulan. En este respecto, Barreras (2004) afirma por ejemplo que:

Es importante conocer las características del alumno en esta etapa para saber qué se puede esperar de él y cómo aproximarnos a él. Los niños del último curso de la segunda etapa [cuarto] y los de la tercera etapa de educación primaria [quinto y sexto] se caracterizan por una serie de rasgos. (2004: 64)

No obstante, Barreras (2004) hace un énfasis especial únicamente sobre la importancia de enseñar vocabulario que designe conceptos $u$ objetos concretos y que resulten familiares para el alumno, dejando así atrás especificaciones sobre la adquisición de otros contenidos o destrezas en dicho tramo de edad. Además, estas apreciaciones ya habían sido puestas de relieve anteriormente por otros autores de renombre como, por ejemplo, Scott e Ytreberg (1990). Tampoco se ofrecen propuestas didácticas concretas, es decir, actividades que ejemplifiquen o materialicen cómo transmitir el contenido léxico considerado como adecuado para los ciclos segundo y tercero. Por lo tanto, estas carencias en la investigación tan solo enfatizan más y más la necesidad de objetivar el proceso de análisis del contenido de los libros de texto para determinar 
con mayor grado de precisión la tipología concreta de actividades que ya existe para abordar la enseñanza del ILE en la Escuela Primaria y destapar los posibles puntos débiles. En otras palabras, estudios como el presente trabajo contribuirían a concretar qué actividades existen ya en los libros de texto para mejorarlos desde un punto de vista cognitivo o, al menos, para que los profesores adopten una postura crítica en la selección de actividades docentes y no den así continuidad a desajustes en cuanto al nivel del contenido y el tramo de edad en el que se encuentran los alumnos. Las propuestas didácticas llevadas a cabo por diversos autores (Halliwell 2000; Brewster et al. 2002; Hearn y GarcésRodríguez 2003; Pérez y Roig 2004; Carol 2007) deberían ser de utilidad para frenar la continuidad de prácticas que promuevan una metodología docente contradictoria con la idiosincrasia (en la dimensión de cognición del lenguaje) de los alumnos a quienes se dirigen dichos materiales y para cada uno de los tramos de edad en la Escuela Primaria. Así pues, los docentes deberían adquirir una postura crítica en el ámbito de la metodología de la enseñanza de lenguas extranjeras para poder apreciar desajustes en la concepción de los tres ejes básicos que componen un método concreto (Sánchez 2009): los principios sobre los que se sustenta un método (eje 1), los contenidos que se promueven (eje 2) y los ejercicios que el libro de texto oferta empleado en el aula oferta para materializar la práctica lingüística (eje 3). Para ejemplificar este argumento, cabría recordar que, si la enseñanza del ILE en la Escuela Primaria en España está enmarcada dentro del Método Comunicativo, el fin último de aprender el idioma sería alcanzar un dominio fluido del mismo. Teniendo en cuenta los contenidos establecidos en el currículo académico para dichos tramos de edad (RDL 126/2014, de 28 de febrero: 40-45) y el equilibrio de actividades (atendiendo a la tipología explícita, implícita y mixta) que se ha hallado en base al análisis de la muestra de libros de texto, encontraríamos fácilmente un conflicto metodológico. Quizás no se esté enfatizando la comunicación (y/o la fluidez comunicativa) sobre otros aspectos de carácter lingüístico como, por ejemplo, la adecuación gramatical o el contenido léxico. En consecuencia, sería necesario practicar una reflexión crítica con el fin de promover directrices que a su vez ejerzan un cambio o una mejora en la presencia de actividades (en base a su tipología) en los libros de texto para la enseñanza y el aprendizaje del ILE en la Escuela Primaria en España.

Si bien es cierto que a lo largo de la década pasada la presencia de actividades para la enseñanza exclusiva de las formas parece haber disminuido en los libros de texto, no existe un patrón detectable que refleje una adaptación en el tipo y número de actividades al desarrollo cognitivo de los niños. Esta tendencia contrastaría 
claramente con los argumentos presentes en la literatura especializada, los cuales abogan por un uso mayor de actividades comunicativas y una presencia menor de actividades enfocadas estrictamente a la enseñanza de las formas de la lengua extranjera. No obstante, la mayoría de los estudios llevados a cabo muestran una serie de debilidades metodológicas que podrían comprometer la validez interna y externa de sus hallazgos (p. ej., número de participantes, tarea, instrumento de recogida de datos, entre otros).

Exceptuando uno de los estudios revisados (Goorhuis-Brouwer y de Bot 2010), la mayoría carecen de una muestra sólida y representativa de informantes, además de medidas fiables y validadas para la recogida de datos y el análisis (p. ej., Ebbels 2007; Kang 2008; Lázaro-Ibarrola y Azpilicueta-Martínez 2014; Montijano-Cabrera 2014; Azkarai y Oliver 2016). Por ejemplo, mientras que en el estudio de Goorhuis-Brouwer y de Bot (2010) más de 300 sujetos realizaron un examen estandarizado y altamente fiable, los otros trabajos parecen mostrar una validez del constructo bastante pobre, con datos obtenidos de muestras informantes más reducidas (cf. Ebbels 2007; Kang 2008, con tres participantes y uno, respectivamente).

El proceso de selección de tareas puede haber constituido otra amenaza para la validez de algunos estudios. Por ejemplo, en dos trabajos similares enfocados al examen de la interacción entre los estudiantes, se implementó un juego de adivinanzas con el fin de observar qué estrategias de negociación del significado eran las elegidas por los estudiantes (Lázaro-Ibarrola y Azpilicueta-Martínez 2014; Azkarai y Oliver 2016). En este último, las autoras reconocían incluso el desequilibrio en la selección de actividades. Por último, otros estudios como el de Montijano-Cabrera (2014) no están verificados empíricamente.

En cuanto a los resultados, la mayoría de las investigaciones sobre la enseñanza del ILE en la escuela primaria informan sobre la carencia de evidencia empírica que sostenga la utilidad del Aprendizaje Integrado de Contenidos y Lenguas Extranjeras (AICLE) para la mejora de ciertas destrezas, como el desarrollo de vocabulario (Agustín-Llach 2015) o destrezas de interacción efectivas (Azkarai y Oliver 2016), en estados tempranos del aprendizaje. Esto sugiere que los aprendices jóvenes que se expongan más a la enseñanza implícita puede que no siempre obtengan mejores resultados que aquellos que reciben instrucción tanto explícita como implícita. En otros países de Europa, sin embargo, los resultados de aprendizaje entre estudiantes inscritos a programas de AICLE tienden a ser más efectivos (Goorhuis-Brouwer y de Bot 2010). De hecho, Agustín-Llach (2015) indica que factores como una edad temprana junto con un nivel de dominio 
bajo de los aprendices informantes en los estudios revisados puede que hayan distorsionado la percepción acerca de los hallazgos.

Lo que parece cierto es que la edad, el ambiente en el aula y las actividades constituyen tres factores clave en la enseñanza y aprendizaje del ILE en la escuela primaria. Dado que la mayor parte de las investigaciones realizadas -a mi saber- no han tratado la dimensión de la tipología y el potencial pedagógico de las actividades en el aula, el presente estudio pretendía cubrir ese hueco. Este estudio debería motivar a otros investigadores para que busquen herramientas más válidas y fiables para la selección de las actividades en futuros trabajos.

\section{Conclusiones e implicaciones pedagógicas}

Las actividades tienen un papel fundamental en el contexto de la enseñanza y aprendizaje del ILE en la escuela primaria. Dado que los profesores son los responsables del uso de las mismas, tanto la selección de actividades como la manera en que se implementan son aspectos cruciales en la investigación. Además, existen implicaciones pedagógicas y cognitivas derivadas del uso de las actividades en entornos de instrucción. Es por ello que los profesores de ILE no deberían ignorar las características idiosincráticas de los diferentes tipos de actividades $\mathrm{y}$, en especial, en los estadios más tempranos de la educación, con estudiantes jóvenes. Ser capaces de identificar el potencial cognitivo de enseñanza de las actividades podría ser el punto de partida para mejorar las intervenciones docentes y así poder obtener resultados más óptimos. En base a esta justificación, el objetivo del presente estudio exploratorio fue examinar el potencial de enseñanza (explícito, implícito o mixto) de una muestra de 1.952 actividades extraídas de 10 libros de primaria.

Tras el análisis se observó una tendencia dispar y asistemática en cuanto a la inclusión de actividades con diferente carga cognitiva, lo cual conlleva que las actividades de carácter explícito tienen a veces un protagonismo injustificado en el ámbito de la enseñanza del ILE en la escuela primaria. Esta tendencia, en todo caso, podría no estar en consonancia con las necesidades pedagógicas de los aprendices más jóvenes. Este hallazgo sugiere que los diseñadores de materiales docentes puede que no sean totalmente conscientes de las técnicas y estrategias más efectivas para ellos. De hecho, la presencia considerable de actividades enfocadas a la enseñanza explícita puede ser evidencia suficiente como para afirmar que los libros de texto deberían contener una proporción de actividades más adecuada 
a las necesidades de los estudiantes de primaria. Estudios como éste deberían ser tomados en cuenta por los diseñadores de materiales didácticos con el fin de mejorar la proporción de actividades enfocadas -parcial o exclusivamente- a la enseñanza de las formas.

Entre algunas de las líneas de investigación futuras estarían: (a) el procesamiento de una muestra mayor de actividades para ser analizadas desde el punto de vista del potencial cognitivo (explícito, implícito o mixto), (b) el estudio de las tendencias en cuanto a la tipología de actividades en las categorías mayormente explícito, mayormente implícito y de potencial mixto, y (c) el análisis de la motivación en relación al uso de diferentes tipos de actividades en el aula.

En cuanto a las implicaciones pedagógicas, existe la necesidad de formar a los profesionales de la enseñanza en el ámbito de la detección y el análisis del potencial cognitivo de las actividades. Es importante que, en todo momento, los profesores sean conscientes de las implicaciones cognitivas derivadas del uso de una actividad u otra en el aula (Freeman 1989; Kelly 2017). El objetivo último sería que sus acciones pedagógicas no interfirieran con la forma idiosincrática que los niños tienen de adquirir el conocimiento. También se podría argumentar que los profesores deberían adoptar una actitud más crítica con los materiales docentes que manejan. Los profesores deberían ser conscientes también de los posibles resultados en el aprendizaje derivados de diferentes intervenciones docentes y del uso de distintas actividades. Así mismo, se deberían desarrollar la capacidad de elegir, filtrar o incluso descartar actividades presentes en el libro de texto, teniendo en cuenta variables como la edad o el perfil del alumno. Por último, una implicación pedagógica positiva derivada del buen uso de las actividades sería la creación de un mayor número de oportunidades para la exposición al input de calidad.

\section{Bibliografía}

AGUSTÍN-LLACH, M. (2015). “Age and type of instruction (CLIL vs. traditional EFL) in lexical development". International Journal of English Studies 16 (1): 75-96.

ALCÓN-SOLER, E. y GARCIA-MAYO, M. (2008). "Incidental focus on form and learning outcomes with young foreign language classroom learners" en Second Language Acquisition and the Younger Learner: Child's Play? (Eds. J. Philp, R. Oliver y A. Mackey). Amsterdam: John Benjamins: 173-192. ALIZADEH, K. (2011). "The advantages of active English teaching, emphasizing at active teaching to EFL child students". Modern Journal of Language Teaching Methods 1 (1): 80-87. 
AZKARAI, A. y IMAZ-AGIRRE, A. (2015). "Negotiation of meaning strategies in child EFL mainstream and CLIL settings". TESOL Quarterly 50 (4): 844-870.

AZKARAI, A. y OLIVER, R. (2016). "Negative feedback on task repetition: ESL vs. EFL child settings". The Language Learning Journal 1-12. https:// doi.org/10.1080/09571736.2016.1196385.

BAARS, B. (1997). "In the Theatre of Consciousness: The Workspace of the Mind”. Journal of Consciousness Studies 4 (4): 292-309.

BARRERAS, M. A. (2004). "Vocabulario y edad: Pautas para su enseñanza en las clases de inglés de educación primaria". Aula Abierta 84: 63-84.

BIALYSTOK, E. (2001). "Metalinguistic aspects of bilingual processing". Annual Review of Applied Linguistics 21 (1): 169-181.

BIALYSTOK, E. (2007). "Cognitive complexity and attentional control in the bilingual mind". Child Development 70 (3): 636-644.

BIALYSTOK, E., MARTIN, M. y VISWANATHAN, M. (2005). "Bilingualism across the lifespan: The rise and fall of inhibitory control". International Journal of Bilingualism, 9 (1): 103-119.

BREWSTER, J., ELLIS, G. y GIRARD, D. (2002). The Primary English Teacher's Guide. Harmondsworth: Penguin.

CAMERON, L. (2001). Teaching Languages to Young Learners. Cambridge: Cambridge University Press.

CAROL, R. y SOBERÓN, A. (2004). Bugs 1 (Student Book). London: Macmillan.

CAROL, R. y SOBERÓN, A. (2004). Bugs 2 (Student Book). London: Macmillan.

CAROL, R. y SOBERÓN, A. (2005). Bugs 5 (Student Book). London: Macmillan.

CAROL, R. y SOBERÓN, A. (2009). Bugs World 2 (Student Book). London: Macmillan.

CEKAITE, A. (2008). "Developing conversational skills in a second language. Language learning affordances in a multiparty classroom setting" en Second Language Acquisition and the Younger Learner: Child's Play? (Eds. J. Philp, R. Oliver y A. Mackey). Amsterdam: John Benjamins: 105-129.

CHARRINGTON, M., COVILL, C. y SHIPTON, P. (2014). Great Explorers 2 (Workbook). Oxford: Oxford University Press.

CORBETT, J. y O'FARRELL, R. (2011). Quest 2 (Student Book). London: Macmillan.

CORBETT, J. y O'FARRELL, R. (2011). Quest 3 (Workbook). London: Macmillan.

CORBETT, J. y O'FARRELL, R. (2012). Quest 4 (Workbook). London: Macmillan. 
COUNCIL OF EUROPE (2001). Common European framework of reference for languages: learning, teaching, assessment. Cambridge: Cambridge University Press.

COVILL, C., CHARRINGTON, M. y SHIPTON, P. (2011). Explorers 2 (Workbook). Oxford: Oxford University Press.

CRIADO, R. (2010). "The Impact of Activity Sequencing on the Differences between ELT Methods: A Critical Analysis of Sample Units”. Porta Linguarum 14 (1): 7-28.

CRIADO, R. y SÁNCHEZ, A. (2009). "The Universal Character of the DEC $->$ PRO Cognitive Sequence in Language Learning and Teaching Materials". Revista Española de Lingüística Aplicada (RESLA) 22: 89-106.

CRIADO, R., SÁNCHEZ, A. y CANTOS, P. (2010). "An Attempt to Elaborate a Construct to Measure the Degree of Explicitness and Implicitness in ELT Materials". International Journal of English Studies 10 (1): 103-129.

DEKEYSER, R. (2015). "Skill Acquisition Theory" en Theories in Second Language Acquisition (Eds. B. VanPatten y J. Williams). Nueva York: Routledge: 94-112.

DEKEYSER, R. y CRIADO, R. (2013). "Automatization, Skill Acquisition, and Practice in Second Language Acquisition" en The Encyclopedia of Applied Linguistics (Ed. C. A. Chapelle). Oxford: Wiley Blackwell: 1-5. https://doi. org/10.1002/9781405198431.wbeal0067.

EBBELS, S. (2007). "Teaching grammar to school-aged children with specific language impairment using Shape Coding". Child Language Teaching and Therapy 23 (1): 67-93.

ELLIS, R. (2009). Implicit and Explicit Knowledge in Second Language Learning, Testing and Teaching. Bristol: Multilingual Matters.

ELLIS, R. (2015). "Form-focused instruction and the measurement of implicit and explicit L2 knowledge" en Implicit and Explicit Learning of Languages (Ed. P. Rebuschat). Amsterdam: John Benjamins: 417-442.

ESCRIBANO, T. y VENTOSA, R. (2006). Sunshine (Student Book). Harlow: Pearson Longman.

ESCRIBANO, T. y VENTOSA, R. (2006). Sunshine (Workbook). Harlow: Pearson Longman.

ESPAÑA. Real Decreto-ley 126/2014, de 28 de febrero, por el que se establece el currículo básico de la Educación Primaria [Internet]. Boletín Oficial del Estado, 1 de marzo de 2014, núm. 52: 2014-2222. <https:// www.boe.es/buscar/pdf/2014/BOE-A-2014-2222-consolidado.pdf> (Acceso 18 noviembre 2018). 
EVANS, S., SHIPTON, P. y TORRES, S. (2014). Great Explorers 4 (Workbook). Oxford: Oxford University Press.

FREEMAN, D. (1989). "Teacher training, development and decision making: A model of teaching and related strategies for language teacher education". TESOL Quarterly 23 (1): 27-45.

GOLDSTEIN, B. y JONES, C. (2015). Smart Planet 1 (Student Book). Cambridge: Cambridge University Press.

GOO, J., GRANENA, G., YILMAZ, Y. y NOVELLA, M. (2015). "Implicit and explicit instruction in L2 learning: Norris y Ortega (2000) revisited and updated" en Implicit and Explicit Learning of Languages (Ed. P. Rebuschat). Amsterdam: John Benjamins: 443-482.

GOORHUIS-BROUWER, S. y DE BOT. K. (2010). "Impact of early English language teaching on L1 and L2 development in children in Dutch schools". International Journal of Bilingualism 14 (3): 289-302.

GRIS-ROCA, J. (2015). "Categorization of activities used in English as a foreign language: A corpus based study with pedagogical materials". Procedia Social and Behavioural Sciences 198: 165-173.

GRIS-ROCA, J. (2017). "Grammar in primary school EFL textbook activities: A corpus-driven study of their teaching nature and related cognitive implications". Research in Corpus Linguistics 5 (1): 57-72.

HALLIWELL, S. (2000). Teaching English in the Primary Classroom. New York: Longman.

HEARN, I., y GARCÉS-RODRÍGUEZ, A. (2003). Didáctica del inglés para Primaria. Madrid: Pearson Educación.

HOUSE, S. (2011). Didáctica del inglés. Classroom Practice. Barcelona: Graó.

HULSTIJN, J. (2002). "Towards a unified account of the representation, processing and acquisition of second language knowledge". Second Language Research 18: 193-223.

HULSTIJN, J. (2005). "Theoretical and Empirical Issues in the Study of Implicit and Explicit Second-language Learning". Studies in Second Language Acquisition 27: 129-140.

KANG, D. (2008). "The classroom language use of a Korean elementary school teacher: Another look at TETE". System 36: 214-226.

KELLY, C. 2017. "The brain studies boom: Using neuroscience in ESL/EFL teacher training" en Innovative Practices in Language Teacher Education (Eds. T. Gregersen y P. D. Maclntyre). Berlin: Springer: 79-99.

LÁZARO-IBARROLA, A. y AZPILICUETA-MARTÍNEZ， R. (2014). "Investigating negotiation of meaning in EFL children with very low levels of proficiency". International Journal of English Studies 15 (1): 1-21. 
LEOW, R. (2015). Explicit Learning in the L2 Classroom: A Student-Centered Approach. Nueva York, NY: Routledge.

MALPAS, S. (2012). Islands 2 (Student Book). London: Pearson.

MALPAS, S. (2012). Islands 2 (Workbook). London: Pearson.

MOHAMED, S. (2013). Big Surprise 6 (Workbook). Oxford: Oxford University Press.

MONTIJANO-CABRERA, M. (2014). Textbook use training in EFL teacher education. Ultrecht Studies in Language and Communication 27: 267-286.

ORMEROD, M. y SHAW, D. (2007). Find Out 3 (Student Book). London: Macmillan.

ORMEROD, M. y SHAW, D. (2007). Find Out 3 (Workbook). London: Macmillan. PAPIOL, E. y TOTH, M. (2005). Bugs 6 (Workbook). London: Macmillan.

PARADIS, M. (2009). Declarative and Procedural Determinants of Second Languages. Amsterdam: John Benjamins.

PÉREZ, P. y ROIG, V. (2004). Enseñar y Aprender inglés en educación infantil y primaria. Barcelona: Horsori.

PHILLIPS, S. y REDPATH, P (2008). Incredible English 5 (Student Book). Oxford: Oxford University Press.

PHILLIPS, S. y REDPATH, P (2008). Incredible English 5 (Workbook). Oxford: Oxford University Press.

PHILliPS, S. y SHIPTON, P. (2011). Explorers 5 (Student Book). Oxford: Oxford University Press.

PHILLIPS, S. y SHIPTON, P. (2014). Great Explorers 6 (Workbook). Oxford: Oxford University Press.

PHILLIPS, S. y SHIPTON, P. (2014). Oxford Rooftops 3 (Workbook). Oxford: Oxford University Press.

PHILLIPS, S. y SHIPTON, P. (2015). Great Explorers 6 (Student Book). Oxford: Oxford University Press.

PHILP, J., OLIVER, R. y MACKEY, A. (2008). Second Language Acquisition and the Younger Learner: Child's Play? Amsterdam: John Benjamins.

READ, C. (2007). 500 Activities for the Primary Classroom. Oxford: Macmillan. READ, C. (2009). Footprints 3 (Student Book). London: Macmillan.

READ, C. (2009). Footprints 3 (Workbook). London: Macmillan.

READ, C. (2009). Footprints 4 (Student Book). London: Macmillan.

READ, C. (2009). Footprints 4 (Workbook). London: Macmillan.

READ, C., y ORMEROD, M. (2013). Team Tiger 4 (Workbook). London: Macmillan.

REBUSCHAT, P. (2015). Implicit and Explicit Learning of Languages. Amsterdam: John Benjamins. 
REILLY, V. (2013). Big Surprise 5 (Student Book). Oxford: Oxford University Press.

SÁNCHEZ, A. (2009). La enseñanza de idiomas en los últimos cien años. Madrid: SGEL.

SCOTT, W. y YTREBERG, L. (1990). Teaching English to Children. London y Nueva York: Longman.

SHAW, D. y ORMEROD, M. (2007). Find Out 2 (Workbook). London: Macmillan.

SHAW, D. y RAMSDEN, J. (2014). High Five! 1 (Workbook). London: Macmillan.

VAN DEN BRANDEN, K. (2008). "Negotiation of meaning in the classroom. Does it enhance reading comprehension?" en Second Language Acquisition and the Younger Learner. Child's Play? (Eds. J. Philp, R. Oliver y A. Mackey). Amsterdam: John Benjamins: 149-169.

WHITE, J. (2008). "Speeding up acquisition of his and her: Explicit L1/L2 contrasts help" en Second Language Acquisition and the Younger Learner. Child's Play? (Eds. J. Philp, R. Oliver y A. Mackey). Amsterdam: John Benjamins: 193-228.

WORRALL, A. (2004). English Adventure 3 (Student Book). Harlow: Pearson Longman.

WORRALL, A. (2004). English Adventure 3 (Workbook). Harlow: Pearson Longman.

WRIGHT, L. (2008). "Home-school connections for international adoptees: repetition in parent-child interaction" en Second Language Acquisition and the Younger Learner. Child's Play? (Eds. J. Philp, R. Oliver y A. Mackey). Amsterdam: John Benjamins: 279-301.

ZANATTA, T. (2007). Mega Zoom 3 (Student Book). London: Richmond Publishing. 